\title{
Plasmonic Refractive Index Sensor and Plasmonic Bandpass Filter Including Waveguide and Four Teeth Based On Fano Resonances
}

\section{Vahid Najjari}

University of Mazandaran

Saeed Mirzanejhad ( $\nabla$ saeed@umz.ac.ir)

University of Mazandaran https://orcid.org/0000-0002-0717-819X

Amin Ghadi

University of Mazandaran

\section{Research Article}

Keywords: Surface plasmon-polaritons, Fano resonances, MIM waveguide, Refractive index sensor, Plasmonic filter

Posted Date: January 3rd, 2022

DOI: https://doi.org/10.21203/rs.3.rs-1055234/v1

License: (c) (i) This work is licensed under a Creative Commons Attribution 4.0 International License. Read Full License 


\title{
Plasmonic Refractive Index Sensor and Plasmonic Bandpass Filter including Waveguide and Four Teeth based on Fano Resonances
}

\author{
Vahid Najjari, Saeed Mirzanejad"'Amin Ghadi
}

Department of Atomic and molecular physics, Faculty of Sciences, Mazandaran University, Babolsar, Iran

E-mail: saeed@umz.ac.ir*

\begin{abstract}
A plasmonic refractive index sensor including a Metal-Insulator-Metal waveguide (MIM) with four teeth is proposed. Transmittance (T), Sensitivity (S) and Figure of Merit (FOM) investigated numerically and analysed via Finite Difference Time Domain method (FDTD). The simulation results show the generation of double Fano resonances in the system that the resonance wavelength and the resonance line-shapes can be adjusted by changing the geometry of the device. By optimizing the structure in the initial configuration, the maximum sensitivity of $1078 \mathrm{~nm} / \mathrm{RIU}$ and FOM of $3.62 \times 10^{5}$ is achieved. Then change the structure parameters. In this case, the maximum sensitivity and FOM are $1041 \mathrm{~nm} / \mathrm{RIU}$ and $2.94 \times 10^{4}$ respectively, thus two detection points can be used for the refractive index sensor. Due to proper performance and adjustable Fano resonance points, this structure is significant for fabricating sensitive refractive index sensor and plasmonic bandpass filter.
\end{abstract}

Keywords Surface plasmon-polaritons . Fano resonances . MIM waveguide . Refractive index sensor . Plasmonic filter

\section{Introduction}

Fano resonance is a resonant scattering phenomenon that occurs during the coupling of a discrete state with a continuous state [1]. Fano resonance can also be caused by the interaction between a wide bright mode and a narrow dark mode [2]. Compared to Lorentzian resonance, this phenomenon has an asymmetric and ultra-sharp transmission spectrum that is very sensitive to change in structure parameters and surrounding environment [3]. These features make it useful for designing measurement tools and using two parameters Sensitivity and FOM (Figure of Merit), the system performance can be evaluated [4]. Since the Fano resonance is strongly influenced by changes in the environment, the use of Fano resonance points in sensors, improves their resolution and sensitivity [5].

Surface Plasmon-polaritons (SPPs) are the result of a coupling between the incident electromagnetic waves and the free electrons of metal surface, which are strongly confined near the metal-dielectric boundary and propagate at the metal-dielectric interface [6, 7]. SPPs have attracted attention of many researchers, and the construction of a variety of devices such as sensors [8], couplers [9], filters [10], demultiplexers [11], slow Light [12] and optical switches [13] have been suggested. In addition, SPPs can be used to transfer data in high-density photonic integrated circuits [14]. Metal-Insulator-Metal (MIM) waveguide coupled resonators have unique properties such as overcome the diffraction limit [15], high wavelength propagation range, simplicity of construction [16] and high ability to confine light at the scale below the wavelength. [17]. In these structures, by merging the properties of integrated electronic (high miniaturization) and photonic (high speed), a unique device can be produced for the next generation, which will bring a very high processing speed [16, 18]. Therefore, more and more scientists are emphasizing research on plasmonic materials and promoting the use of these materials in the production and development of new tools [19]. The use of plasmonic materials to produce optically integrated circuits has been considered by many researchers due to the high ability of these structures to confine light in the sub-wavelength range [20]. Recently, various plasmonic structures have been proposed to study Fano resonances, and various types of plasmonic sensors have been reported based on this resonance. For example, Jing Guo et al. Designed a refractive index nanosensor based on Fano resonance, which includes an MIM waveguide and a single rectangular cavity without a long side with a maximum sensitivity of $1840 \mathrm{~nm} / \mathrm{RIU}$ [21]. Yihong Fang et al. Have introduced a plasmonic sensor 
refractive index with multi-channel Fano resonances with a sensitivity of $1940 \mathrm{~nm} / \mathrm{RIU}$ [22]. A refractive index sensor designed by Mengmeng Wang et al. Includes a baffle coupled with a circular split ring resonance cavity with a sensitivity of $1114.3 \mathrm{~nm} / \mathrm{RIU}$ [23]. The refractive index sensor is based on Fano resonances in plasmonic waveguide with two-way ring resonators designed by Xuewei Zhang et al. With a sensitivity of $1160 \mathrm{~nm} / \mathrm{RIU}$ [24]. In all papers, scientists have made every effort to ensure that the structure, while simple in design, has the best performance in terms of sensitivity and Figure of Merit.

In this paper, we propose a refractive index sensor with a simple plasmonic structure consisting of a waveguide with four teeth. The transmission spectrum, and magnetic field distributions $\left|\mathrm{H}_{\mathrm{z}}\right|$ are simulated by the two-dimensional FDTD method with PML absorber boundary conditions and simulation parameters, $\Delta \mathrm{x}$ and $\Delta \mathrm{y}$ are equal to $5 \mathrm{~nm}$. Due to the importance of FOM and sensitivity values in the refractive index sensor, both cases are investigated and the results are given. Simplicity in construction and high performance can lead to the use of the proposed structure as a refractive index sensor.

\section{Simulation and analysis}

Simulations and analyzes are performed in two dimensions to avoid the complexities of data processing. Fig. 1 shows a two-dimensional design for the proposed structure consisting of a waveguide with four teeth, including three teeth at the top and one tooth below the waveguide. In this structure, the width of the waveguide and all the teeth are equal to $\mathrm{w}$ and four teeth with lengths L1, L2 and h1, h2 are assumed. According to Fig. 1, the distance between the teeth is assumed $\mathrm{d} 1$ and $\mathrm{d} 2$. To ensure that the single-mode spectrum propagates through the waveguide and the four teeth, the value of $\mathrm{w}$ is assumed to be a constant value of $50 \mathrm{~nm}$.

According to Fig. 1, the electromagnetic spectrum enters the device from the left and exits from the right, so the $\mathrm{P}_{\text {in }}$ is the power at the input port and the $\mathrm{P}_{\text {out }}$ is the power at the output port. The parameters are calculated at the two points $\mathrm{P}_{\text {in }}$ and $\mathrm{P}_{\text {out }}$ and the transmittance $(\mathrm{T})$ is defined as $\mathrm{T}=\mathrm{P}_{\text {out }} / \mathrm{P}_{\text {in }}$. In the initial configuration, in order to design the best performance of the device and make it easier to study the characteristics of the transmission spectrum, L1 and L2 are assumed to be equal to $205 \mathrm{~nm}$. The size of h1 is $75 \mathrm{~nm}$ and $\mathrm{h} 2$ is $105 \mathrm{~nm}$. According to the results of several simulations, the best performance of the device is obtained for equal distance between the teeth, and therefore the values $\mathrm{d} 1$ and $\mathrm{d} 2$ are considered equal and with a length of $15 \mathrm{~nm}$. The air

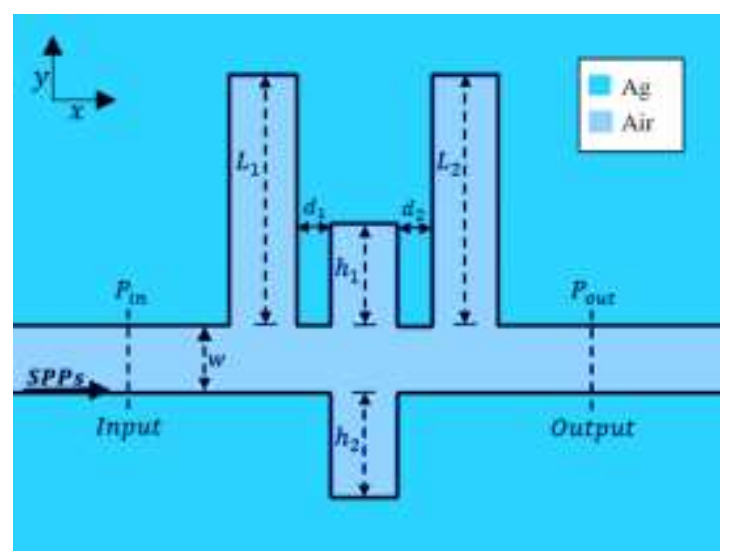

Fig. 1 Proposed structure for plasmonic sensor and filter

and silver sections are shown in Fig. 1, where the air dielectric constant is 1.0 and the permeability of the silver metal follows the Drude model [25]:

$\varepsilon=\varepsilon_{\infty}-\frac{\omega_{p}{ }^{2}}{\omega^{2}+i \omega \gamma}$

where the dielectric constant at the infinite frequency is taken as $\varepsilon_{\infty}=3.7$, the plasmonic oscillation frequency is taken as $\omega_{\mathrm{p}}=9.1 \mathrm{eV}$ and the characteristic collision frequency is $\gamma=0.018 \mathrm{eV}$. The wave reflection due to the addition of teeth to the waveguide changes the amplitude and phase of the wave propagated inside the waveguide and therefore changes the transmission spectrum line-shape and creates a sharp and asymmetric line-shape compared to the toothless state. The optical responses of the structure and distribution of the $\left|\mathrm{H}_{\mathrm{z}}\right|$ simulated and calculated by the two-dimensional FDTD method with the PML absorber boundary conditions. The purpose of using PML is to absorb waves inside the structure and prevent the impact of unwanted reflection waves in the simulation environment. Using standing wave theory, the resonant wavelength can be calculated in terms of effective refractive index [26]:

$\lambda_{m}=\frac{2 \operatorname{Re}\left(n_{e f f}\right) L}{m-\varphi / \pi}, m=1,2,3, \ldots$

the phase shift $\Phi$ is due to the reflection of light from the metal-air interface in the resonant cavity, and $\mathrm{L}$ is the effective length of the resonant cavities. $m$ is the order of resonant mode, which is an integer. Also, $\operatorname{Re}\left(\mathrm{n}_{\mathrm{eff}}\right)$ is the real part of the refractive index and $\mathrm{n}_{\text {eff }}$ is defined as follows:

$n_{e f f}=\sqrt{\left[\varepsilon_{m}+\left(\frac{k}{k_{0}}\right)^{2}\right]}$ 


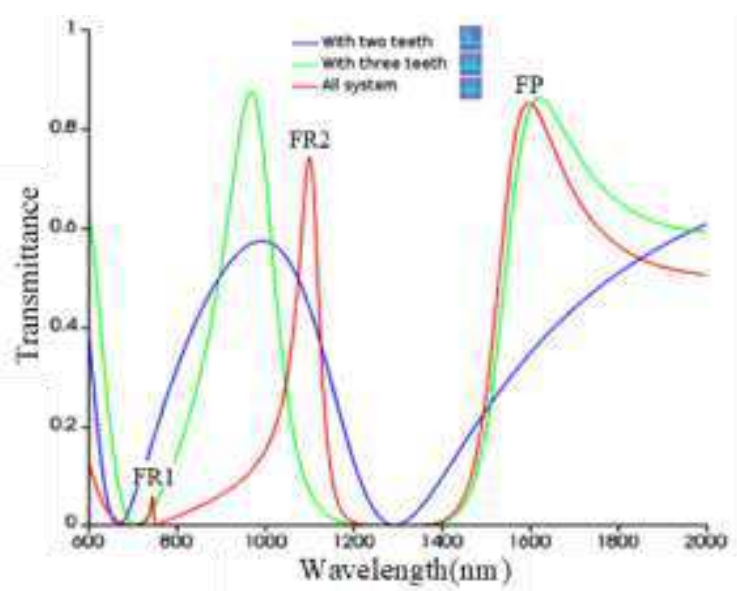

Fig. 2 Transmission spectrum of the plasmonic structure with two teeth, three teeth and the entire device. The parameters are set as $\mathrm{L} 1=205 \mathrm{~nm}, \mathrm{~L} 2=205 \mathrm{~nm}, \mathrm{~W}=50 \mathrm{~nm}, \mathrm{~h} 1=75 \mathrm{~nm}, \mathrm{~h} 2=105 \mathrm{~nm}, \mathrm{~d} 1=15 \mathrm{~nm}$ and $\mathrm{d} 2=15 \mathrm{~nm}$

where $\mathrm{k}$ is the wave vector in the waveguide. In order to understand the effect of adding teeth to the waveguide on the performance of the device, the transmission spectra for the structure with two teeth, three teeth and the entire structure are drawn(see Fig. 2). As shown in Fig. 2, the addition of teeth to waveguide creates resonance peaks in the
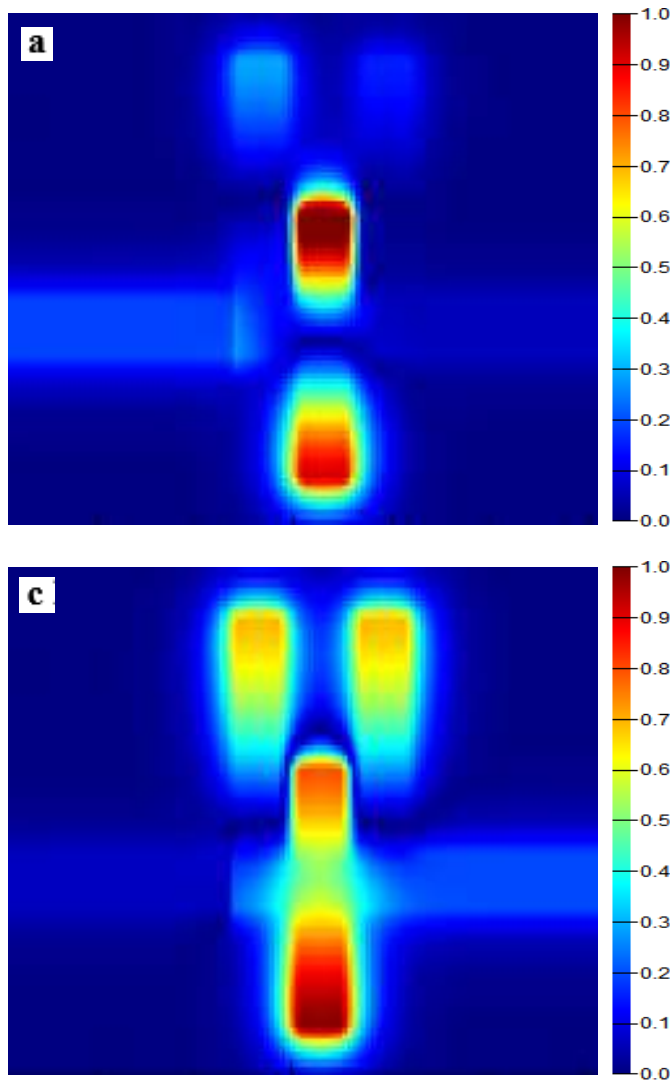

transmission line, and when the structure is entirely simulated, it has two Fano resonance points at FR1 and FR2 which are located at $\lambda=743 \mathrm{~nm}$ and $\lambda=1100 \mathrm{~nm}$, respectively. Also, the transmission spectrum has resonance point at $\lambda=1597 \mathrm{~nm}$, the line shape of which is asymmetric but not very sharp, and is marked with the name FP, which is not investigated in this work. In other words, points FR1 and FR2 will be investigated for sharper peaks and better properties. In order to better understand the internal mechanism of the transmission spectrum, the field distribution $\left|\mathrm{H}_{\mathrm{z}}\right|$ in resonance peaks and dips studied. As shown in Fig. 3, SPPs are inserted through the $p_{\text {in }}$ port and then propagated in the waveguide, and when the wavelength reaches to resonance point, most of them are coupled to the teeth. According to the Figs. 3a to $3 d$, field distribution $\left|H_{z}\right|$, showing that in which part of the structure the electromagnetic field is concentrated. In Fig. 3a, the electromagnetic field $\left|\mathrm{H}_{z}\right|$ plotted at $\lambda=744 \mathrm{~nm}$, which is the peak of FR1, and it is clear that the field is concentrated in teeth $\mathrm{h} 1$ and h2. This indicates that Fano resonance in FR1 is the result of an interaction between the narrow resonance caused by the h1 and h2 teeth and the wide resonance caused by the MIM. It is important to noted that according to Fig. 3a, only by changing the size of these two teeth can
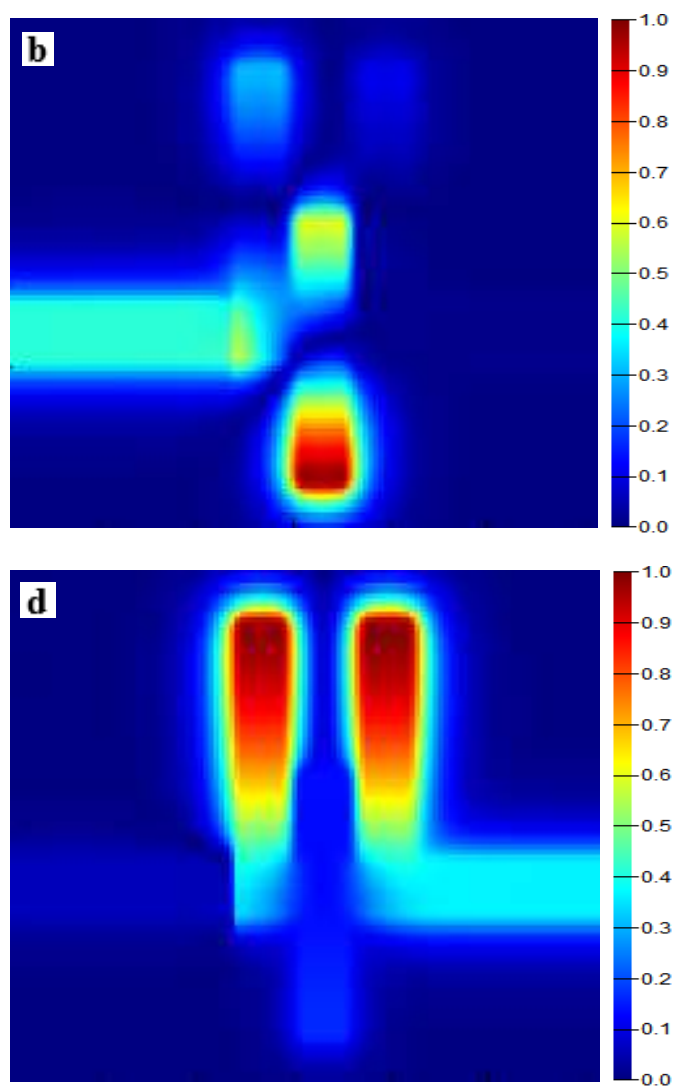

Fig. 3 Display of field distribution $\left|\mathrm{H}_{\mathrm{z}}\right|$ In structure at the resonance wavelength $\mathbf{a} \lambda=744 \mathrm{~nm}, \mathbf{b} \lambda=753 \mathrm{~nm}, \mathbf{c} \lambda=1100 \mathrm{~nm}$ and $\mathbf{d} \lambda=1597 \mathrm{~nm}$ 
controlled the transmittance and the resonant wavelength in FR1. According to the above, it can be claimed that FR2 is also the result of the narrowing resonance of the teeth and the wide resonance of MIM and the FP point is also the result of the resonance of the teeth with lengths L1 and L2 with wide resonance of MIM [27]. The main purpose of this work is to investigate the characteristics of a plasmonic structure for use as a refractive index sensor. Judgment of sensor performance is possible by two comparable parameters, sensitivity and FOM. Sensitivity(S) is an important parameter for evaluating sensor properties, which is expressed by [28]:

$S=\frac{d \lambda}{d n}(n m / R I U)$

which $d \lambda$ is the amount of change in the resonant wavelength by changing the refractive index $\mathrm{dn}$ due to changes in the device environment. In order to evaluate the performance of the device in different environments, we calculate the value of the transmittance for different refractive indices in which the refractive index is changed from 1.00 to 1.14 . Fig. 4 shows the transmission spectra at different wavelengths by varying the refractive index value. According to Fig. 4, as the refractive index of the environment increases, the shape of the transmission spectrum line remains unchanged. Also, the value of the transmittance at the resonance points did not

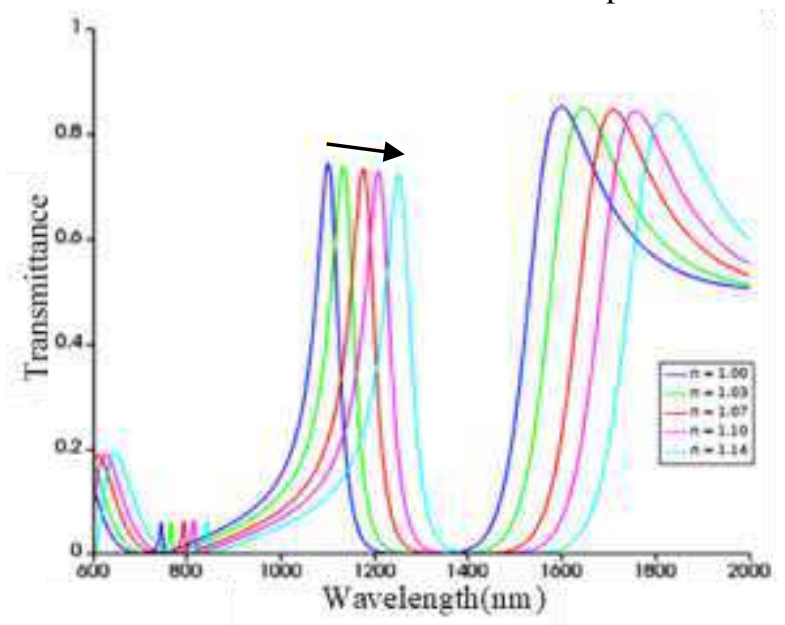

Fig. 4 The transmittance spectra of the structure for different wavelengths by changing the refractive index from 1.00 to 1.14

change much, but the resonant wavelength increased. As shown in Fig. 5a, the Fano resonance wavelength changes linearly by changing the refractive index, which is in accordance with Eq. 2. Using the calculated data and Eq. 4, we investigate the sensitivity at the two Fano resonance wavelength. At point FR1 the sensitivity is $696 \mathrm{~nm} / \mathrm{RIU}$ and at FR2 it is $1078 \mathrm{~nm} / \mathrm{RIU}$. Another important parameter in the (a)

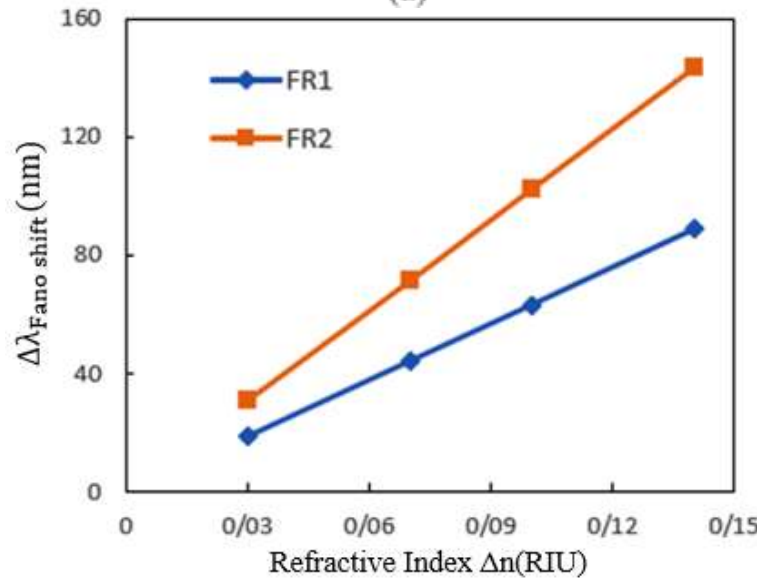

(b)

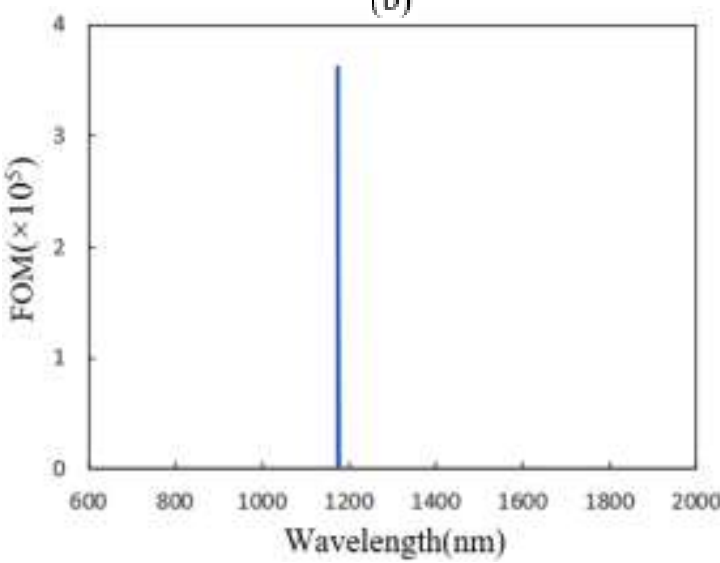

Fig. 5. a Sensitivity and b FOM for the structure with the proposed parameters

evaluation of refractive index nanosensors is Figure of Merit (FOM), which are [29]:

$F O M^{*}=\frac{\Delta T}{T \cdot \Delta n}=\frac{T(\lambda, n)-T\left(\lambda, n_{0}\right)}{T\left(\lambda, n_{0}\right) \cdot \Delta n}$

And

$F O M=\left(F O M^{*}\right)_{\max }$

$\mathrm{T}\left(\lambda, \mathrm{n}_{0}\right)$ is the transmittance in the initial state and $\mathrm{T}(\lambda, \mathrm{n})$ is the transmittance after changing the environment refractive index. FOM represents the change in the intensity of the relative transmission spectrum $\Delta \mathrm{T} / \mathrm{T}$ due to the change in the refractive index $(\Delta \mathrm{n})$, which is calculated at wavelength $\lambda_{0}$. According to Eq. 5 and the available data, the FOM for FR1 was 482.17 , but for FR2 it was $3.62 \times 10^{5}$, which is shown in Fig. 5b. According to the sensitivity and FOM values, in the structure, the FR2 resonance point has a better performance and can be used as a detection point in the index sensor. To compare the performance of the proposed device with other 


\begin{tabular}{|c|c|c|c|}
\hline Reference & Year & S(nm/RIU) & FOM \\
\hline$[2]$ & 2020 & 1075 & $9.95 \times 10^{4}$ \\
\hline$[30]$ & 2020 & 540 & 101.3 \\
\hline$[6]$ & 2020 & 886.16 & - \\
\hline$[31]$ & 2020 & - & 269 \\
\hline$[32]$ & 2018 & 780 & $1.56 \times 10^{5}$ \\
\hline$[33]$ & 2018 & 825 & $5.47 \times 10^{4}$ \\
\hline$[34]$ & 2017 & 1057 & - \\
\hline$[35]$ & 2016 & 938 & $1.35 \times 10^{4}$ \\
\hline$[36]$ & 2016 & 750 & 53.7 \\
\hline$[37]$ & 2016 & 596 & - \\
\hline$[38]$ & 2013 & 530 & 650 \\
\hline This work & 2021 & 1078 & $3.62 \times 10^{5}$ \\
\hline
\end{tabular}

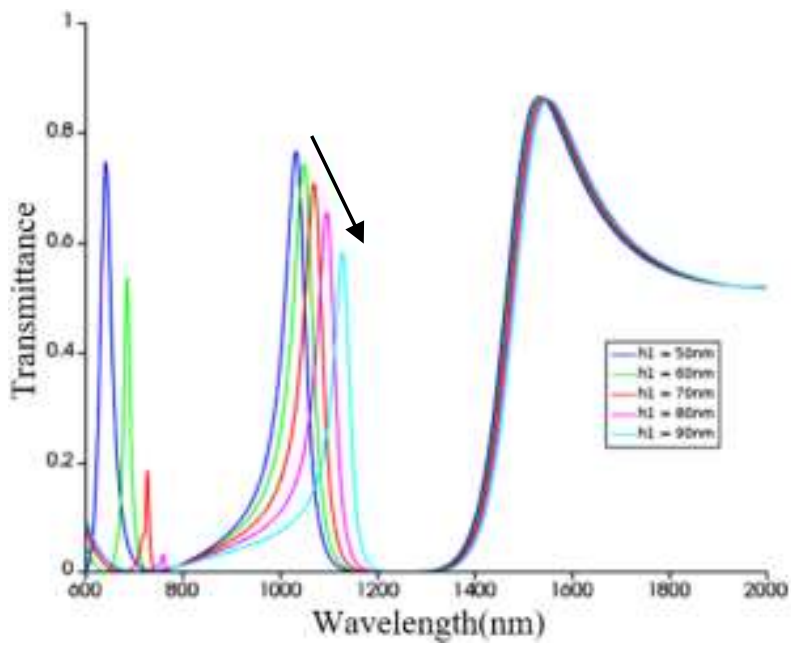

Fig. 7 The influence of change h1 on transmission properties if other structure parameters are fixed

in other words, these changes can affect the performance of the device. Now, in order to study the effect of changing the structure size on the device performance, we change the size of the teeth and investigate its effect on the transmittance, sensitivity and FOM, which ultimately leads to determining

Table 1. Comparison of the performance of the proposed sensor and other structures performed

structures, table 1 shows the sensitivity and FOM values of some of the structures previously proposed and this work. As

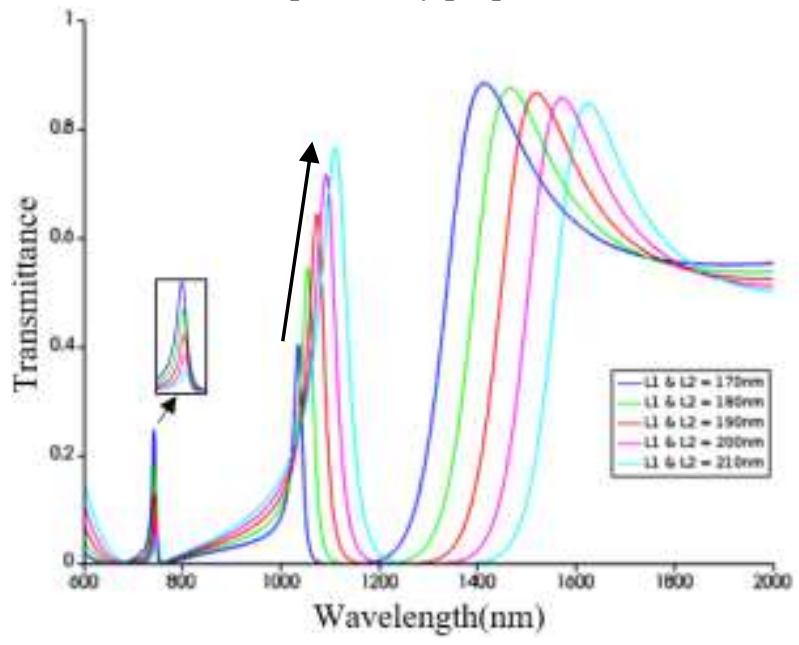

Fig. 6 The influence of change L1 and L2 on transmission properties if $h 1$ and $h 2$ are fixed

can be seen, despite the simplicity of the proposed structure design in this work, the sensor has better performance and the sensitivity than other works. Although the proposed structure is in nanoscale, a change in the size of its components can affect the sensitivity and FOM values, and

Fig. 8 The influence of change h2 on transmission properties if other structure parameters are fixed

the acceptable error rate in making the device as a refractive index sensor so that the operation of the device is not disturbed. For this purpose, we first fixed the sizes $\mathrm{h} 1$ and $\mathrm{h} 2$ to $75 \mathrm{~nm}$ and $105 \mathrm{~nm}$ respectively and change $\mathrm{L} 1$ and $\mathrm{L} 2$ from $170 \mathrm{~nm}$ to $210 \mathrm{~nm}$ with $10 \mathrm{~nm}$ step. Fig. 6 shows the changes in the transmission spectra for equal and simultaneous changes in L1 and L2 sizes. As shown in Fig. 6, with increasing L1 and L2, the transmittance line-shape is fixed but the transmission in FR2 increases while for the point 
FR1, this change Decreases the transmission value. In this case, the transmittance at the FP point does not change significantly, but the resonant wavelength increases(redshift). For system optimization, we assume L1 and L2 to be fixed and equal to $195 \mathrm{~nm}$, and investigate the effect of changing h1 and h2 on the transmission spectra. Now, we change h1 from $50 \mathrm{~nm}$ to $90 \mathrm{~nm}$ in $10 \mathrm{~nm}$ steps. As shown in Fig. 7, increasing the length of h1 reduces transmittance in FR1 and FR2 points. Then, According to Fig. 8, by fixing L1 and L2 equal to $195 \mathrm{~nm}$ and $\mathrm{h} 1$ equal to $80 \mathrm{~nm}$, we assume the h2 with lengths of 50,60, 70, 80 and 90nm. As shown, decreasing $\mathrm{h} 2$ leads to an increase in the spectrum transmittance in FR1 and FR2 and decreases the resonant wavelength(blueshift). Finally, we try to design a structure so that we have the highest sensitivity and FOM at the same time in FR1 and FR2 points. After optimizing the geometry of the structure, L1 and L2 are considered equal to $195 \mathrm{~nm}$, as well as h1 to $80 \mathrm{~nm}$ and $\mathrm{h} 2$ to $75 \mathrm{~nm}$. For the new sizes of the device, the transmission spectra are plotted in Fig. 9 and, as

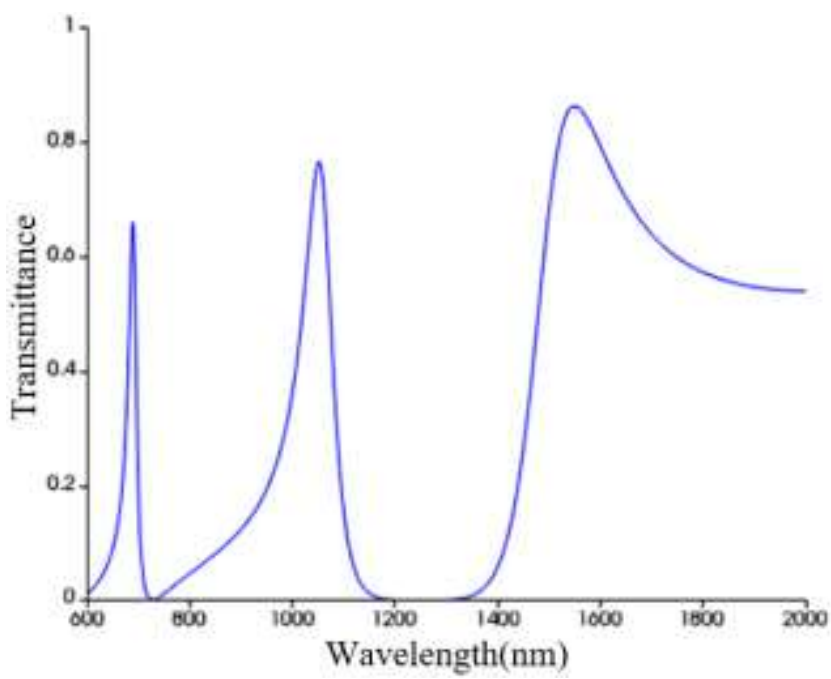

Fig. 9 Transmission spectrum for new plasmonic structure. The parameters are set as $\mathrm{L} 1=195 \mathrm{~nm}, \mathrm{~L} 2=195 \mathrm{~nm}, \mathrm{~W}=50 \mathrm{~nm}, \mathrm{~h} 1=80 \mathrm{~nm}$, $\mathrm{h} 2=75 \mathrm{~nm}, \mathrm{~d} 1=15 \mathrm{~nm}$ and $\mathrm{d} 2=15 \mathrm{~nm}$

can be seen, two Fano resonance points are generated at wavelengths of 704nm (FR1) and 1078nm (FR2). As can be seen, in the second structure, the wavelengths of the Fano resonance points shift to smaller wavelengths. Therefore, by changing the structure parameters, we can adjust the resonance points. We now plot the distribution of the magnetic field $\left|\mathrm{H}_{\mathrm{z}}\right|$ in the wavelengths of $704 \mathrm{~nm}, 737 \mathrm{~nm}$, $1078 \mathrm{~nm}$ and 1613nm as shown in Fig. 10. Again, for the new structure sizes, we change the environment refractive index from 1.00 to 1.14 and plot the transmission spectra as shown in Fig. 11 and investigate sensitivity and FOM values using
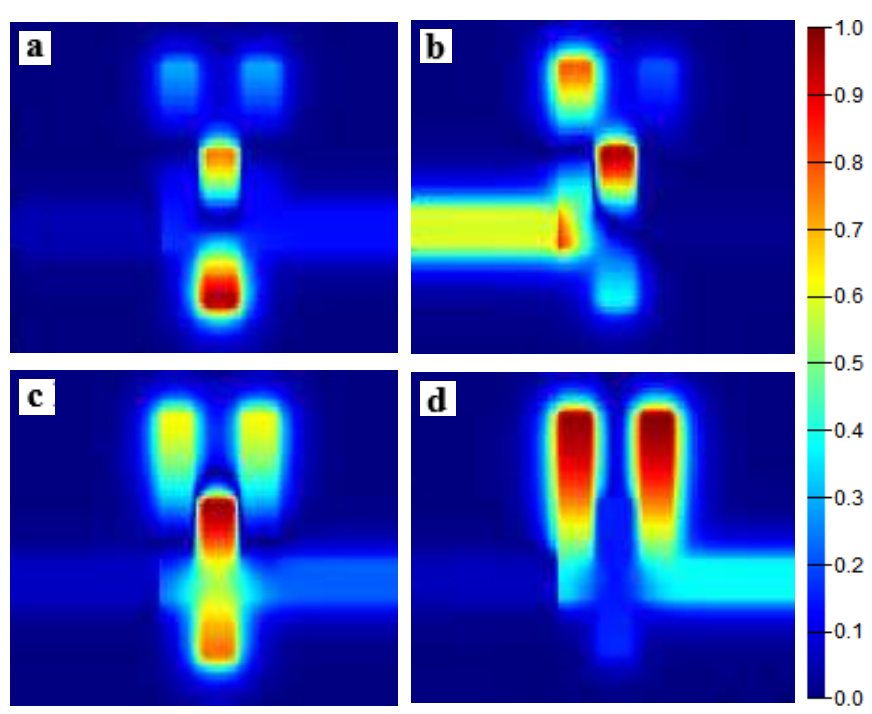

Fig. 10 Display of field distribution $\left|\mathrm{H}_{\mathrm{z}}\right|$ In structure at the resonance wavelength $\mathbf{a} \lambda=704 \mathrm{~nm}, \mathbf{b} \lambda=737 \mathrm{~nm}, \mathbf{c} \lambda=1078 \mathrm{~nm}$ and $\mathbf{d}$ $\lambda=1613 \mathrm{~nm}$

Eqs. 4 and 6. The calculations show that in the new structure, the sensitivity at the FR1 and FR2 resonance points are $659 \mathrm{~nm} / \mathrm{RIU}$ and $1041 \mathrm{~nm} / \mathrm{RIU}$, and FOM are $1.14 \times 10^{3}$ and $2.94 \times 10^{4}$, respectively. In the proposed structure with new sizes, two Fano resonance points with good sensitivity and FOM values have been produced, which can be used to build a high sensitivity refractive index sensor. Also, due to the adjustability of Fano resonance points in structure, this device can be used as a plasmonic bandpass filter.

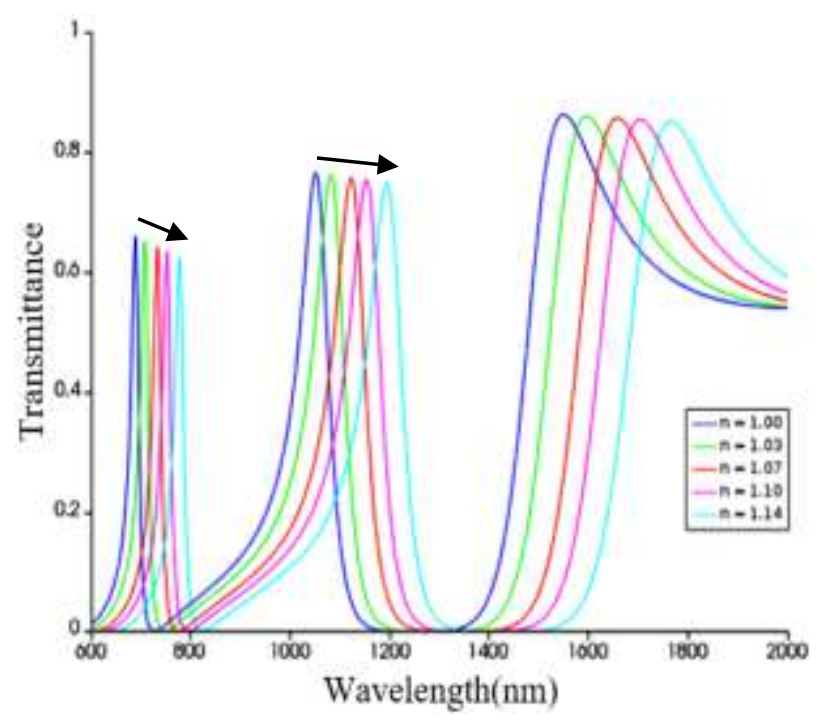

Fig. 11 transmittance spectra of the structure with modified parameters by changing the refractive index 


\section{Conclusion}

In summary, in this work, a refractive index sensor and plasmonic bandpass filter with a simple structure including MIM with four teeth based on SPPs was proposed and simulated and investigated by numerical method FDTD with PML absorption boundary conditions. In the initial configuration, by changing the refractive index of the structure, sensitivity was calculated and the best value was $1078 \mathrm{~nm} / \mathrm{RIU}$ at the FR2 resonance point. Due to the importance of FOM in the sensors, the value of this parameter was also calculated in the same structure and the best value of $3.62 \times 10^{5}$ was obtained for the FR2 resonance point. Also, by changing the structure parameters, the transmittance spectrum line-shape includes two Fano resonance points, which sensitivity of $659 \mathrm{~nm} / \mathrm{RIU}$ and $1041 \mathrm{~nm} / \mathrm{RIU}$ and FOM of $1.14 \times 10^{3}$ and $2.94 \times 10^{4}$ was calculated for FR1 and FR2 points respectively. The most important feature of the structure introduced in this paper is the appropriate sensitivity and FOM and ease of construction. Also, according to the results obtained for sensitivity and FOM, this structure can be used as a sensitive plasmonic refractive index sensor. Also, due to the adjustability of Fano resonance points with changing the structure parameters, this device can be used as a plasmonic bandpass filter.

Data Availability Data sharing is not pplicable to this article as no datasets were generated or analyzed during the current study.

Code Availability Numerical analysis of this paper is based on FDTD method.

\section{Compliance with Ethical Standards}

Competing Interests The authors declare that they have no competing interests.

\section{Funding information}

This study hasn't any financial support.

Authors' Contributions All authors have same contribution in the analytical and numerical calculations and read and approved the final manuscript.

Consent to publish The participant has consented to the submission of the case report to the journal.

Consent to participate Informed consent was obtained from all individual participants included in the study.

Ethical approval Not applicable, because this article does not contain any studies with human or animal subjects.

\section{References}

1. Bianconi A (2002) Ugo Fano and shape resonances, AIP Conference Proceedings, 19th Int. Conference Roma June 2428, 2002

2. Yanga Q, Liua X, Guoa F, Baib H, Zhanga B, Lia X, Tana Y, Zhanga Z (2020) Multiple Fano resonance in MIM waveguide system with cross-shaped cavity. Optik 220:165163. https://doi.org/10.1016/j.ijleo.2020.165163

3. Chen J, Li J, Liu X, Rohimah S, Tian H, Qi D (2021) Fano resonance in a MIM waveguide with double symmetric rectangular stubs and its sensing characteristics. Optics Communications 482:126563

4. Chen Z, Yu L, Wang L, Duan G, Zhao Y, Xiao J (2015) Sharp Asymmetric Line Shapes in a Plasmonic Waveguide System and its Application in Nanosensor. IEEE J Lightwave Technol 33(15):3250-3253

5. Wang J, Liu X, Li L, He J, Fan C, Tian Y, Ding P, Chen D, Xue Q, Liang E (2013) Huge electric field enhancement and highly sensitive sensing based on the Fano resonance effect in an asymmetric nanorod pair. J Opt 15(10):105003

6. F Md. Hassan, Hasan Md M, Ahmed Md I, Sagor R H (2020) Numerical Investigation of a Plasmonic Refractive Index Sensor Based on Rectangular MIM Topology. IEEE 2020 International Seminar on Intelligent Technology and Its Applications (ISITIA) 22-23 July 2020.

7. Barnes W L, Dereux A, T. W. Ebbesen T W (2003) Surface plasmon subwavelength optics. Nature 424(46): 824-830

8. Tsigaridas G N (2017) A Study on Refractive Index Sensors Based on Optical Micro-Ring Resonators. Photonic Sens 7: 217-225. https://doi.org/10.1007/s13320-017-0418-0

9. Wahsheh R A, Lu Z, Abushagur M A G (2009) Nanoplasmonic couplers and splitters. Optics Express 17(21):19033-19040

10. Lai W, Wen K, Lin J, Guo Z, Hu Q, Fang Y (2018) Plasmonic filter and sensor based on a subwavelength end-coupled hexagonal resonator. Applied Optics 57(12):6369-6374

11. Wu Y D (2014) High Transmission Efficiency Wavelength Division Multiplexer Based on Metal-Insulator-Metal Plasmonic Waveguides. IEEE J Lightwave Technol 32(24):4844-4848. https://doi.org/10.1109/JLT.2014.2359938

12. Lu Q, Wang Z, Huang Q, Jiang W, Wu Z, WangY, Xia J (2017) Plasmon-Induced Transparency and High-Performance Slow Light in a Plasmonic Single-Mode and Two-Mode Resonators Coupled System. IEEE J Lightwave Technol 35(9):1710-1717. https://doi.org/10.1109/JLT.2017.2648819

13. Min C, Veronis G (2009) Absorption switches in metaldielectric-metal plasmonic waveguides. Opt Express 17(13):10757-10766

14. Fang Y, Sun M (2015) Nanoplasmonic waveguides: towards applications in integrated nanophotonic circuits. Light Sci Appl 4(e294). https://doi.org/10.1038/lsa.2015.67

15. Xiao G, Xu Y, Yang H, Ou Z, Chen J, Li H, Liu X, Zeng L, Li J (2021) High Sensitivity Plasmonic Sensor Based on Fano Resonance with Inverted U-Shaped Resonator. Sensors 21(4): 1164. https://doi.org/10.3390/s21041164

16. Achi S E, Hocini A, Salah H B, Harhouz A (2020) Refractive Index Sensor MIM Based Waveguide Coupledwith a Slotted Side Resonator. Progress in Electromagnetics Research M 96:147-156. https://doi.org/10.2528/PIERM20061803 
17. Barnes W L, Dereux A, Ebbesen T W (2003) Surface plasmon subwavelength optics. Nature 424:824-830

18. Schuller J A, Barnard E S, Cai W, Jun Y C, White J S, Brongersma M L (2010) Plasmonics for extreme light concentration and manipulation. Nature Materials 9:193-204. https://doi.org/10.1038/nmat2630

19. Su H, Yan S, Yang X, Guo J, Wang J, Hua E (2020) Sensing Features of the Fano Resonance in an MIM Waveguide Coupled with an Elliptical Ring Resonant Cavity. Appl Sci 10(15):5096. https://doi.org/10.3390/app10155096

20. Sorger V J, Oulton R F, Ma R M, Zhang X (2012) Toward integrated plasmonic circuits. MRS Bulletin 37:728-738. https://doi.org/10.1557/mrs.2012.170

21. Guo J, Yang X, Wang Y, Wang M, Hua E, Yan S (2020) Refractive Index Nanosensor with simple Structure based on Fano Resonance. IEEE Photonics Journal 12(4): 4800710. https://doi.org/10.1109/JPHOT.2020.3015988

22. Fang Y, Wen K, Li Z, Wu B, Guo Z (2019) Plasmonic refractive index sensor with multi-channel Fano resonances based on MIM waveguides. Modern Physics Letters B 34(16): 2050173. https://doi.org/10.1142/S0217984920501730

23. Wang M, Zhang M, Wang Y, Zhao R, Yan S (2019) Fano Resonance in an Asymmetric MIM Waveguide Structure and Its Application in a Refractive Index Nanosensor. Sensors 19(4):791. https://doi.org/10.3390/s19040791

24. Zhang X, Qi Y, Zhou P, Gong H, Hu B, Yan C (2018) Refractive Index Sensor Based on Fano Resonances in Plasmonic Waveguide with Dual Side-Coupled Ring Resonators. Photonic Sens 8:367-374.

25. Fang Y, Wen K, Li Z, Wu B, Chen L, Zhou J, Zhou D (2018) Multiple Fano Resonances Based on End-coupled Semi-ring Rectangular Resonator. IEEE photonics journal 11(4): 4801308. https://doi.org/10.1109/JPHOT.2019.2914483

26. Sagor R H, Hassan Md F, Yaseer A A, Surid E, Ahmed Md I (2020) Highly sensitive refractive index sensor optimized for blood group sensing utilizing the Fano resonance. Appl Nano sci 11:521-534.

27. Wang Y, Yu S, Zhao T, Hu Z, Wang S (2020) High figure of merit refractive index nanosensor based on Fano resonances in waveguide. Journal of Nanophotonics 14(2):026021. https://doi.org/10.1117/1.JNP.14.026021

28. Yu S, Zhao T, Yu J, Pan D (2019) Tuning Multiple Fano Resonances for On-Chip Sensors in a Plasmonic System. Sensors 19(7):1559. https://doi.org/10.3390/s19071559

29. Wen K, Hu Y, Chen L, Zhou J, Lei L, Guo Z (2015) Fano Resonance with Ultra-High Figure of Merits Based on Plasmonic Metal-Insulator-Metal Waveguide. Plasmonics 10:27-32. https://doi.org/10.1007/s11468-014-9772-6

30. Haffar R El, Farkhsi A, Mahboub O (2020) Optical properties of MIM plasmonic waveguide with an elliptical cavity resonator. Applied Physics A 126:486. https://doi.org/10.1007/s00339-020-03660-w

31. Zhu J, Lou J (2020) High-sensitivity Fano Resonance Temperature Sensor in MIM Waveguides Coupled with a Polydimethylsiloxane-sealed Semi-square Ring Resonator. Results in Physics 18:103183.

32. Qiao L, Zhang G, Wang Z, Fan G, Yan Y (2018) Study on the fano resonance of coupling M-type cavity based on surface plasmon polaritons. Optics Communications 433:144-149

33. Chen Y, Luo P, Liu X, Di Y, Han S, Cui X, He L (2018) Sensing performance analysis on Fano resonance of metallic double-baffle contained MDM waveguide coupled ring resonator. Optics and Laser Technology 101:273-278

34. Zhang Z D, Wang R B, Zhang Z Y, Tang J, Zhang W D, Xue C Y, Yan S B (2017) Electromagnetically Induced Transparency and Refractive Index Sensing for a Plasmonic Waveguide with a Stub Coupled Ring Resonator. Plasmonics 12:1007-1013. https://doi.org/10.1007/s11468-016-0352-9

35. Binfeng Y, Hu G, Zhang R, Yiping C (2016) Fano resonances in a plasmonic waveguide system composed of stub coupled with a square cavity resonator. Journal of Optics 18(5):055002.

36. Pang S, Huo Y, Xie Y, Hao L (2016) Fano resonance in MIM waveguide structure with oblique rectangular cavity and its application in sensor. Optics Communications 381:409-413

37. Zhang Z, Luo L, Xue C, Zhang W, Yan S (2016) Fano Resonance Based on Metal-Insulator-Metal WaveguideCoupled Double Rectangular Cavities for Plasmonic Nanosensors. Sensors 16(5):642. https://doi.org/10.3390/s16050642

38. Qiang C Z, Wei Q J, Jing C, Dong L Y, Qiang H Z, Qiang L W, Jun X J, Qian S (2013) Fano Resonance Based on Multimode Interference in Symmetric Plasmonic Structures and its Applications in Plasmonic Nanosensors. Chinese Physics Letters 30(5):057301 